02,13

\title{
Сверхпроводниковые гетероструктуры с прослойкой из материала с сильным спин-орбитальным взаимодействием
}

\author{
(C) Г.А. Овсянников ${ }^{1}$, А.С. Гришин ${ }^{1}$, К.И. Константинян ${ }^{1}$, А.В. Шадрин ${ }^{1,2}$, \\ A.М. Петржик ${ }^{1}$, Ю.В. Кислинский ${ }^{1}$, G. Cristiani $^{3}$, G. Logvenov ${ }^{3}$ \\ ${ }^{1}$ Институт радиотехники и электроники им. В.А. Котельникова РАН, \\ Москва, Россия \\ ${ }^{2}$ Московский физико-технический институт, \\ Долгопрудный, Россия \\ ${ }^{3}$ Max Planck Institute for Solid State Research, \\ Stuttgart, Germany \\ E-mail: gena@hitech.cplire.ru
}

\begin{abstract}
Исследуются сверхпроводниковые гетероструктуры с прослойкой из материала, в котором наблюдается сильное спин-орбитальное взаимодействие. В качестве материалов с сильным спин-орбитальным взаимодействием нами выбраны иридаты стронция, имеющие Ruddlesden-Popper-структуру $\left(\operatorname{Sr}_{n+1} \operatorname{Ir}_{n} \mathrm{O}_{3 n+1}\right.$; $n=1,2, \infty)$ : парамагнитный металл $\mathrm{SrIO}_{3}$ и магнитный изолятор $\mathrm{Sr}_{2} \mathrm{IrO}_{4}$. C помощью лазерной абляции были получены эпитаксиальные пленки этих материалов, а также гетероструктуры на основе эпитаксиальных пленок купратного сверхпроводника $\mathrm{YBa}_{2} \mathrm{Cu}_{3} \mathrm{O}_{x}$. Для электрофизических измерений изготавливались мезаструктуры микронных размеров с верхним электродом из бислоя $\mathrm{Au} / \mathrm{Nb}$.
\end{abstract}

Работа выполнена при частичной поддержки программой Президиума РАН.

DOI: 10.21883/FTT.2018.11.46651.18NN

\section{1. Введение}

Оксиды $5 d$-переходных металлов вызывают значительный интерес из-за возникновения в них сильного спин-орбитального взаимодействия $\left(E_{S O}\right)$, которое сосуществует наряду с электрон-электронным взаимодействием $(U)$. Эта комбинация, как предсказано теоретически [1], позволяет реализовать несколько новых квантовых состояний вещества, таких как топологический мотовский изолятор [2,3], материалы, в которых реализуется квантовый спиновый эффект Холла и квантовый аномальный эффект Холла [4-6], вейлевский полуметалл [7] и даже высокотемпературный сверхпроводник [8]. В $5 d$-переходных металлах $E_{S O} \sim 0.4 \mathrm{~V}$ в несколько раз превышает $E_{S O} 3 d$-переходных металлов. $E_{S O}$ оксидов с $5 d$-переходными металлами сравнима с энергией электронных корреляций $U \sim 0.5 \mathrm{eV}$. Ряд экзотических квантовых состояний наблюдаются в Ruddleden-Popper-серии оксидов иридия $\left(\operatorname{Sr}_{n+1} \operatorname{Ir}_{n} \mathrm{O}_{3 n+1}\right.$; $n=1,2$, и $\infty)$ [1-4]. Это семейство демонстрирует сильную зависимость физических параметров материалов от размерности системы. Слоистый $\mathrm{Sr}_{2} \mathrm{IrO}_{4}(n=1)$ является двумерным антиферромагнитным моттовским изолятором с расщеплением зон $J_{e f f}=1 / 2$ [9], $\mathrm{Sr}_{3} \mathrm{Ir}_{2} \mathrm{O}_{7}$ $(n=2)$ является плохим магнитным изолятором с температурой ферромагнитного перехода $285 \mathrm{~K}$ [10], a $\mathrm{SrIrO}_{3}(n \rightarrow \infty)$ - плохой парамагнитный металл. Иридаты становятся изоляторами, когда кристаллическое поле [11] расщепляет вырожденные состояния $5 d$-электронов на $e_{g}$ и $t_{2 g}$ зоны, а частично заполненная $t_{2 g}$ зона расщепляется на $J_{e f f}=3 / 2$ и $J_{e f f}=1 / 2$ зоны за счет сильного спин-орбитального взаимодействия ионов иридия. При этом появление моттовской щели в
$J_{e f f}=1 / 2$ зоне, вызванной кулоновским взаимодействием, становится энергетически выгодным. В ячейке $\mathrm{SrIrO}_{3}$ с атомом иридия соседствуют шесть атомов кислорода, в ячейке $\mathrm{Sr}_{2} \mathrm{IrO}_{4}$ - только четыре. С увеличением числа слоев $\mathrm{IrO}_{2}$ (увеличением $n$ ) увеличивается энергетическая ширина проводимости Ir $5 d$-состояний $(\mathrm{W})$ и как результат $\mathrm{SrIrO}_{3}$ становится коррелированным парамагнитным плохим металлом [1,9].

В данной работе представлены результаты структурных и электротранспортных исследований пленок $\mathrm{SrIrO}_{3}$ и $\mathrm{Sr}_{2} \mathrm{IrO}_{4}$, выращенных на четырех монокристаллических подложках (001) $\mathrm{SrTiO}_{3} \quad$ (STO), (001) $\left(\mathrm{LaAlO}_{3}\right)_{3}+\left(\mathrm{Sr}_{2} \mathrm{AlTaO}_{6}\right)_{0.7}$ (LSAT), (110) $\mathrm{NdGaO}_{3}$ (NGO) и (001) $\mathrm{LaAlO}_{3}$ (LAO), кристаллические параметры которых задают величину деформации кристаллической решетки пленки.

\section{2. Методика эксперимента и экспериментальные образцы}

Тонкие пленки иридатов наносились на монокристаллические подложки методом лазерной абляции мишени. Мишени изготавливались из смеси порошков $\mathrm{SrCO}_{3}$ и $\mathrm{IrO}_{2}$ в соотношении, обеспечивающем состав $\mathrm{Sr}_{1.85} \mathrm{IrO}_{4}$. После перемешивания полученный порошок прессовался в таблетки. Затем проводилась термообработка при высокой температуре с промежуточным измельчением и повторным прессованием. Рентгенофазовый анализ показал, что полученные мишени являются однофазными и имеют тетрагональную кристаллическую решетку: пространственная группа $I 4 / \mathrm{mmm}$, параметры решетки: 
$a=0.3884 \mathrm{~nm}, c=1.2891 \mathrm{~nm}[12]$. Полученные параметры близки к табличным значениям для монокристаллов, которые составляют соответственно $0.3888 \mathrm{~nm}$ и $1.290 \mathrm{~nm}$ в работе [13]. Измерения СКВИД магнетометром показывают переход мишени в антиферромагнитное состояние при $240 \mathrm{~K}$, а пересчитанная из магнитного момента величина намагниченности составляет $0.014 \mu_{B} / \mathrm{Ir}$ (рис. 1). Наиболее близкое значение $0.023 \mu_{B} / \operatorname{Ir}$ дается в обзоре [14], нейтронные исследования [15] дают на порядок более высокие значения. Температура перехода в антиферромагнитное состояние $\left(T_{N}=240 \mathrm{~K}\right)$ совпадала во всех рассмотренных работах. Таким образом, учитывая близкие к табличным параметры кристаллической решетки и температуры Нееля синтезированной мишени, можно считать, что изготовленная мишень действительно имела состав $\mathrm{Sr}_{2} \mathrm{IrO}_{4}$.

Напыление пленок производилось с помощью $\mathrm{KrF}$ эксимерного лазера в атмосфере кислорода и (или) аргона при температуре подложки от $770-730^{\circ} \mathrm{C}$. Температура подложки контролировалась пирометром, частота импульсов составляла $2-5 \mathrm{~Hz}$, энергия - более $1 \mathrm{~J} / \mathrm{cm}^{2}$, количество импульсов варьировалось в зависимости от требуемой толщины иридатной пленки. После напыления и охлаждения при атмосфере напыления пленки выдерживались при $500^{\circ} \mathrm{C}$ в атмосфере кислорода в течение $30 \mathrm{~min}$, а затем медленно охлаждались.

Кристаллографические параметры пленок и подложек определялись с помощью четырех кружного рентгеновского дифрактометра, измерением рентгеновских дифрактограмм в режиме $2 \Theta / \omega$ и $\varphi$-сканирования, а также измерением кривых качания.

Первая пленка $\mathrm{Sr}_{2} \mathrm{IrO}_{4}$ была выращена при давлении кислорода 0.05 mbar на подложке (100) $\mathrm{SrTiO}_{3}$. Из рентгеновской дифрактограммы (рис. 2) следует, что дифракционный пик от пленки сильно размыт и наряду с основной $\mathrm{Sr}_{2} \mathrm{IrO}_{4}$ фазой обнаруживается побочная фаза, вероятно состава $\mathrm{SrIrO}_{3}$. Согласно диаграмме роста иридатов, представленной в [16] оптимальной температурой роста эпитаксиальных пленок $\mathrm{Sr}_{2} \mathrm{IrO}_{4}$ является температура $700^{\circ} \mathrm{C}$ при давлении кислорода $10^{-3} \mathrm{mbar}$. Поскольку поддерживать стабильное давление кислорода в районе $10^{-3} \mathrm{mbar}$ практически невозможно при остаточном давлении в камере $10^{-6} \mathrm{mbar}$, кислородная атмосфера была заменена на аргонную. Как видно из (рис. 2), в атмосфере аргона (0.5 mbar) пик от побочной фазы не наблюдается, а $c$-параметр наблюдаемой фазы равен $1.283 \mathrm{~nm}$, что даже хорошо соответствует значениию $1.274 \mathrm{~nm}$ для пленки $\mathrm{Sr}_{2} \mathrm{IrO}_{4}$, выращенной на $\mathrm{SrTiO}_{3}$ подложки [13]. При равной толщине наиболее близкую к объемному монокристаллу структуру имели пленки на подложках (100) $\mathrm{SrTiO}_{3}$ и $(110) \mathrm{NdGaO}_{3} . c$ - параметр таких пленок для подложек (110) $\mathrm{NdGaO}_{3}-1.287 \mathrm{~nm}$.

В тоже время для получения эпитаксиальных пленок $\mathrm{SrIrO}_{3}$ из той же мишени требуется повысить давление кислорода на два порядка выше и уменьшить температуру. Эпитаксиальные пленки $\mathrm{SrIrO}_{3}$ толщиной 30-60 nm были получены методом лазерной абляции

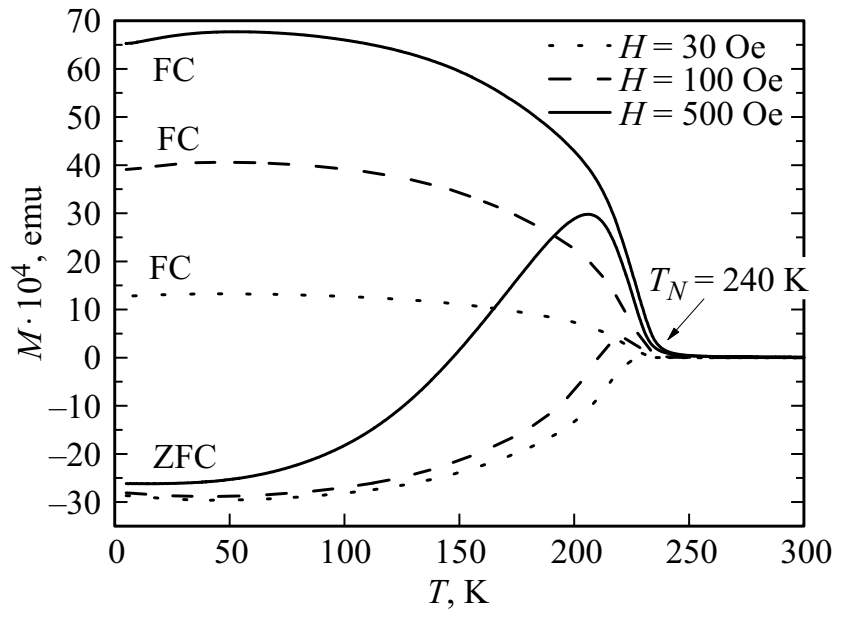

Рис. 1. Зависимость величины магнитного момента мишени $\mathrm{Sr}_{2} \mathrm{IrO}_{4}$ размером $1.5 \times 1 \times 0.5 \mathrm{~mm}^{3}$ от температуры при двух режимах измерений: $\mathrm{FC}$ - заморозка в магнитном поле, ZFC - заморозка в нулевом магнитном поле. Температура перехода в антиферромагнитное состояние $-240 \mathrm{~K}$.

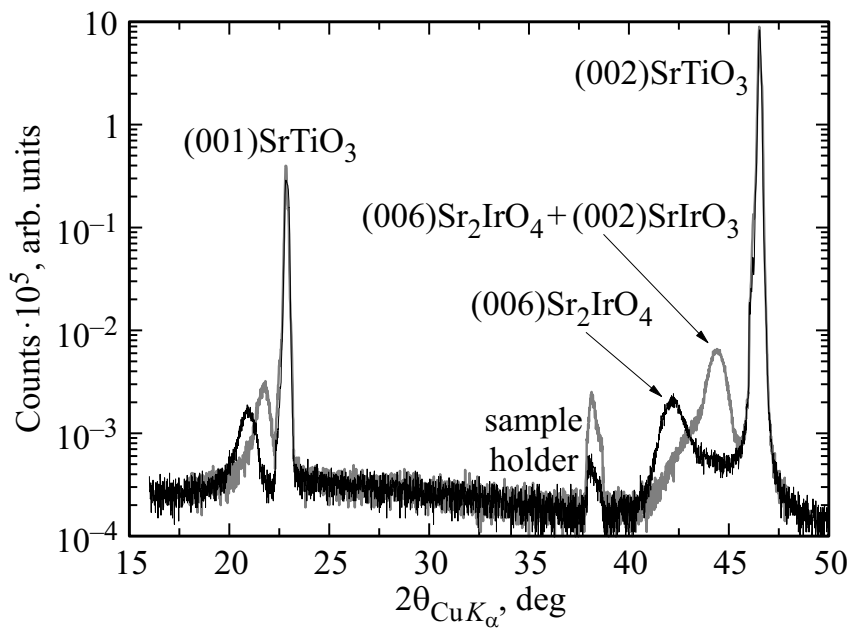

Рис. 2. Рентгеновские дифрактограммы, снятые в режиме бреговского отражения, пленок иридатов, нанесенных методом лазерной абляции на подложку (100) $\mathrm{SrTiO}_{3}$ при атмосфере кислорода $0.05 \mathrm{mbar}$ (серая линия) и в атмосфере аргона $0.5 \mathrm{mbar}$ (черная линия), толщина пленок $17 \mathrm{~nm}$.

на монокристаллических подложках из (001) $\mathrm{SrTiO}_{3}$ (STO), (001) $\mathrm{LaAlO}_{3}+\mathrm{Sr}_{2} \mathrm{AlTaO}_{6}$ (LSAT), (110) $\mathrm{NdGaO}_{3}$ (NGO) и (001) $\mathrm{LaAlO}_{3}$ (LAO) при температуре $770^{\circ}$ и давлении кислорода $0.3 \mathrm{mbar}$ [17].

На $\theta-2 \theta$-дифрактограммах полученных пленок около сильных отражений от подложки имеются отражения от пленки, при этом других отражений от пленки не наблюдается (см. рис. 3). Можно предположить, что пленки имеют структуру близкую к структуре подложек, то есть являются перовскитами либо имеют одну из близких к ней перовскито подобных структур. Это предположение подтверждается снятием полюсных фигур для „косых“ отражений от пленок. Исследование полюсных 


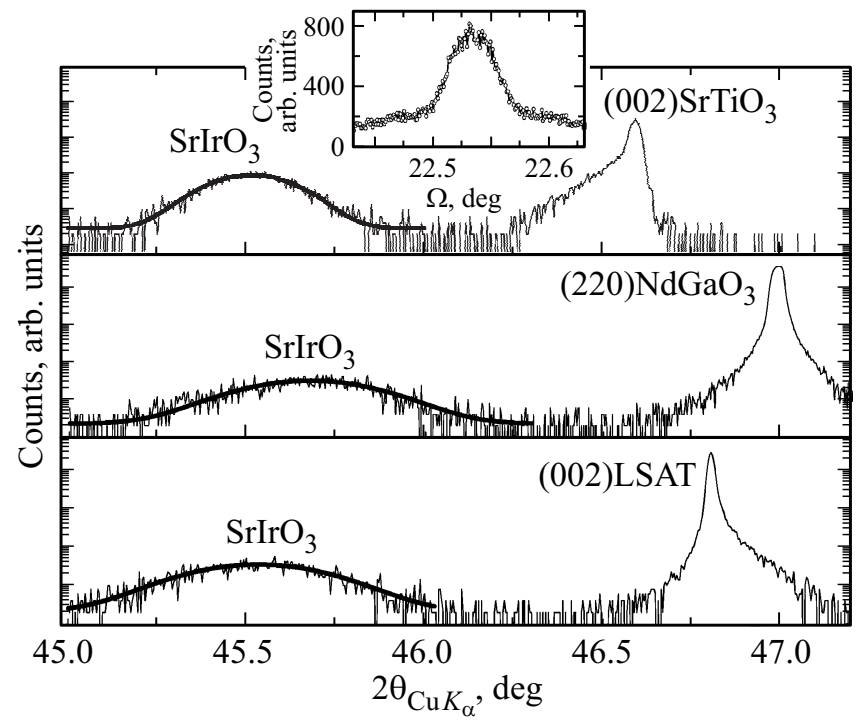

Рис. 3. Рентгеновские дифрактограммы, снятые в режиме бреговского отражения, пленок $\mathrm{SrIrO}_{3}$, выращенных на подложках (001) $\mathrm{SrTiO}_{3}$, (001)LSAT, (110) $\mathrm{NdGaO}_{3}$. На вставке представлена кривая качания для пленки на подложке (001) $\mathrm{SrTiO}_{3}$. Величина ширины кривой качания на полувысоте от максимума $\Delta=0.05^{\circ}$ является минимальной для рассматриваемых в данной статье пленок.

фигур показывает, что структуру пленки на подложке (110) NGO можно описать с помощью перовскита с периодами решетки $a_{f}=b_{f}=c_{f}=0.397 \mathrm{~nm}$. Четыре пика (110)SIO обнаружены при значениях углов $\phi$ равных $0,90,180$ и $270^{\circ}$ при угле $\psi=45^{\circ}$. Пленка растет на подложке по механизму „куб на куб“.

\section{3. Электрофизические параметры тонких пленок иридатов}

Сопротивление пленок изучалось четырехточечным методом, исключающим влияние контактного сопротивления. Контактные площадки изготавливались катодным напылением Pt через металлическую маску.

Были измерены зависимости удельного сопротивления от температуры для пленок иридата стронция, выращенных на подложках LSAT, NGO и STO. Эти подложки обеспечивают малое рассогласование кристаллографических параметров материалов пленки и подложки [18]. Измерялись пленки толщиной $34 \mathrm{~nm}$. Удельные сопротивления пленок при комнатной температуре на несколько порядков превышают значения, представленные в работе [13], что может быть связано с различием дефектности пленок. На рис. $4, b$ представлена зависимость удельного сопротивления от обратной температуры. Линейные участки кривых $\rho(1 / T)$ соответствуют механизму активационной (диэлектрической) проводимости, для которой характерна зависимость $\rho=\rho_{0} \exp \left(\Delta E_{g} / 2 k T\right.$ [13]. Здесь $\Delta E_{g}$ - величина диэлектрической щели, которая для наших пленок составляла
208 . . $240 \mathrm{mV}$, а $k$ - коэффициент Больцмана. Видно, что описанный механизм характерен для температур $100-300 \mathrm{~K}$. Для диапазона более низких температур (выше пунктирной линии на рис. $3, b$ ) наблюдаются утечки в измерительном стенде, вклад которых становится заметен при $R>50 \mathrm{G} \Omega$. При повышении температуры в дополнение к активационному подключается дополнительный механизм проводимости. Предположительно этот механизм является трехмерной проводимостью с переменной длиной прыжка [13], для которого характерна зависимость $\rho=\rho_{0} \varepsilon \xi \pi\left(T_{0} / T\right)^{1 / 4}$. В температурном диапазоне, где данный механизм становится преобладающим, наблюдается линейная зависимость $\rho\left(T^{-1 / 4}\right)$. Радиус прыжка $r$ при минимальной температуре прыжковой проводимости был оценен по формуле $r=a T_{0} / T$, где $a-$ радиус локализации. Радиус прыжка составляет $13 \ldots 17 \mathrm{~nm}$, и не превышает толщин рассматриваемых пленок, что подтверждает возможность возникновения механизма трехмерной прыжковой проводимости для диапазона высоких температур.

На рис. 4 показаны температурные зависимости удельного сопротивления пленок $\mathrm{SrIrO}_{3}$ для четырех подложек. Видно, что наименьшими удельными сопротивлениями обладали пленки $\mathrm{SrIrO}_{3}$, выращенные на подложках STO, которые имеют наиболее близкий к $\mathrm{SrIrO}_{3}$ параметр кристаллической решетки. Сопротивления пленок возрастали с уменьшением параметра подложки (см. рис. 5). Аналогичное изменение удельного сопротивления пленок $\mathrm{SrIrO}_{3}$ для подложек $(001) \mathrm{MgO}$ и (110) $\mathrm{GdScO}_{3}$ представлено в работах $[19,20]$ для подложек с большими параметрами кристаллической решетки.

Удельное сопротивление пленок $\mathrm{SrIrO}_{3} / \mathrm{LAO}$ при комнатной температуре по порядку величины совпадает с удельным сопротивление поликристаллических образцов, полученных при высоком давлении $(2-3 \mathrm{~m} \Omega \cdot \mathrm{cm})[21]$, а сопротивление пленок $\mathrm{SrIrO}_{3} / \mathrm{STO}$ на порядок меньше.

Сопротивление пленок растет с понижением температуры для LSAT, NGO и LAO подложек, а пленки $\mathrm{SrIrO}_{3} / \mathrm{STO}$ имеют перегиб на зависимости $\rho(T)$ при $T_{M I} \approx 210 \mathrm{~K}$. Следовательно $\mathrm{SrIrO}_{3} / \mathrm{STO}$ пленка является металлом при $T>T_{M I}$ и переходит в состояние изолятора при низких температурах.

В работе [22] на подложках $\mathrm{CdScO}_{3}$ с большими параметрами решетки в псевдокубическом представлении $c=0.396 \mathrm{~nm}$ пленки $\mathrm{SrIrO}_{3}$ были металлическими, тогда как температурный ход удельного сопротивления пленок $\mathrm{SrIrO}_{3}$ на подложках $\mathrm{NdGaO}_{3}$ с меньшим параметром $c=0.386 \mathrm{~nm}$ был диэлектрическим. Величина сопротивления удельного сопротивления при комнатной температуре уменьшалась с увеличением параметра $c$, как и в нашем случае.

Отметим, что в диапазоне температур, где наблюдается рост сопротивления, зависимость $\rho(T)$ для пленок $\mathrm{SrIrO}_{3}$ имеет степенной характер, а не экспоненциальный характер как в случае $\mathrm{Sr}_{2} \mathrm{IrO}_{4}$. Для описания температурной зависимости сопротивления пленок $\mathrm{SrIrO}_{3}$ 

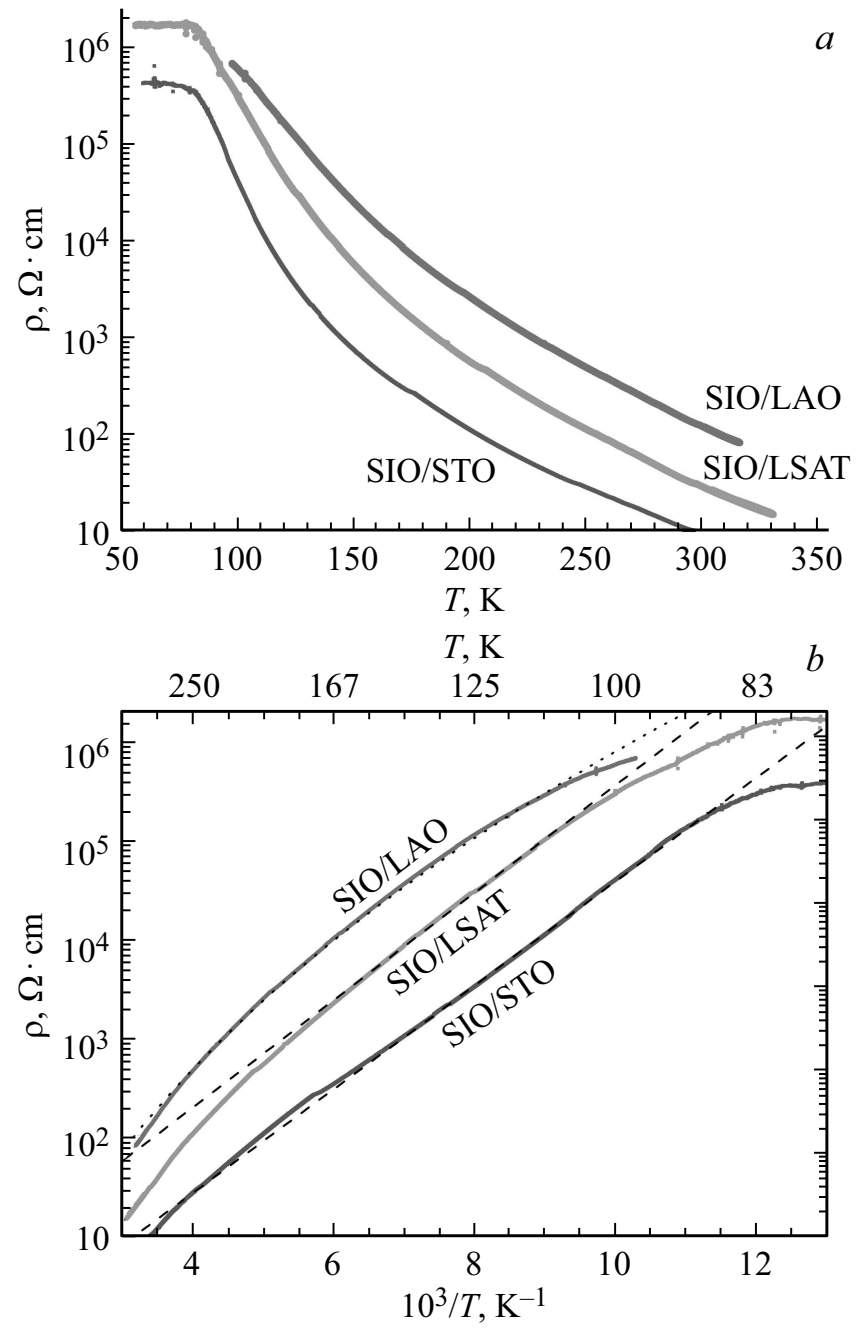

Рис. 4. $a-$ температурные зависимости удельных сопротивлений эпитаксиальных пленок $\mathrm{Sr}_{2} \mathrm{IrO}_{4}$, выращенных на подложках (001) $\mathrm{SrTiO}_{3}$, (001) LSAT, (110) $\mathrm{NdGaO}_{3}$, представленные в полулогарифмическом масштабе; $b-$ зависимость от обратной температуры.

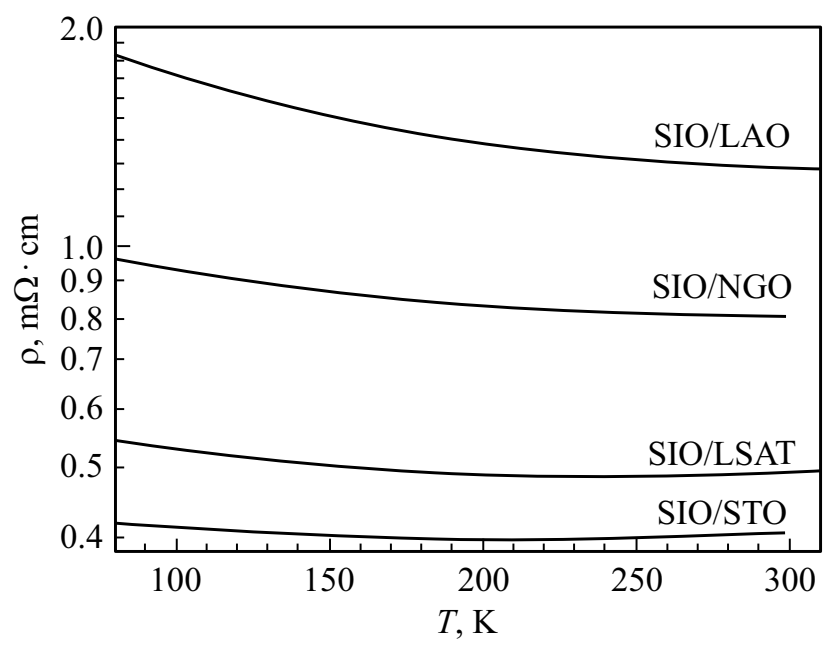

Рис. 5. Температурные зависимости удельных сопротивлений эпитаксиальных пленок $\mathrm{SrIrO}_{3}$ выращенных на подложках: (001) $\mathrm{SrTiO}_{3}$, (001) LSAT, (110) $\mathrm{NdGaO}_{3}$, (001) $\mathrm{LaAlO}_{3}$. использовалась аппроксимация

$$
\rho=\rho_{0}-\alpha T^{3 / 4}+\beta T^{3 / 2},
$$

где $\rho_{0}$ - остаточное сопротивление, $\alpha-$ параметр трехмерной слабой локализации для систем с сильным электронным взаимодействием, $\beta$ - параметр, описывающий неупругое рассеяние из-за взаимодействия электронов с антиферромагнитными магнонами [20,23,24].

Коэффициент, характеризующий слабую локализацию для $\mathrm{SrIrO}_{3} / \mathrm{NGO}$ пленок, $\alpha / \rho_{0}=0.003$ оказывается в два раза меньше, чем для $\mathrm{SrIrO}_{3} / \mathrm{STO}: \alpha / \rho_{0}=0.006$. Возможным механизмом, определяющим локализацию, может быть сдвиг дислокаций, вызванный напряжением пленки, хотя не следует исключать и влияние кислородных вакансий. Необычные электрические транспортные свойства $\mathrm{SrIrO}_{3}$ могут объясняться искажением $\mathrm{IrO}_{6}$ октаэдра, которое в свою очередь приводит к уменьшению перекрытия орбиталей и формированию щели в зоне проводимости. При увеличении рассогласования пленки и подложки уменьшается взаимодействие с антиферромагнитными магнонами: для $\mathrm{SrIrO}_{3} / \mathrm{STO} \beta / \rho_{0}=5 \cdot 10^{-5}$, а для $\mathrm{SrIrO}_{3} / \mathrm{NGO} \beta / \rho_{0}=1 \cdot 10^{-4}$. Для $\mathrm{SrIrO}_{3} / \mathrm{STO}$ пленок температура перехода металл-изолятор оказывается при $T_{0}=210 \mathrm{~K}$, а для $\mathrm{SrIrO}_{3} / \mathrm{NGO}$ - значительно выше: $T_{0} \gg 300 \mathrm{~K}$. Аналогичный переход металл-изолятор происходит в $\mathrm{BaRuO}_{3}[21,25]$.

\section{4. Сверхпроводящие гетероструктуры}

Эпитаксиальная пленка $\mathrm{YBa}_{2} \mathrm{Cu}_{3} \mathrm{O}_{x}$ напылялась непосредственно на подложку при $830^{\circ} \mathrm{C}$ и давлении кислорода $0.5 \mathrm{mbar}$, последующий слой $\mathrm{Sr}_{2} \mathrm{IrO}_{4}$ наносился в атмосфере аргона при давлении $0.5 \mathrm{mbar}$ и температуре $700^{\circ} \mathrm{C}$. После охлаждения камеры до $500^{\circ} \mathrm{C}$ пленка выдерживалась в атмосфере кислорода в течение $30 \mathrm{~min}$. Далее наносился защитный слой золота. Все слои напылялись в одной камере и в одном технологическом цикле. На рис. 6 представлены рентгеновские

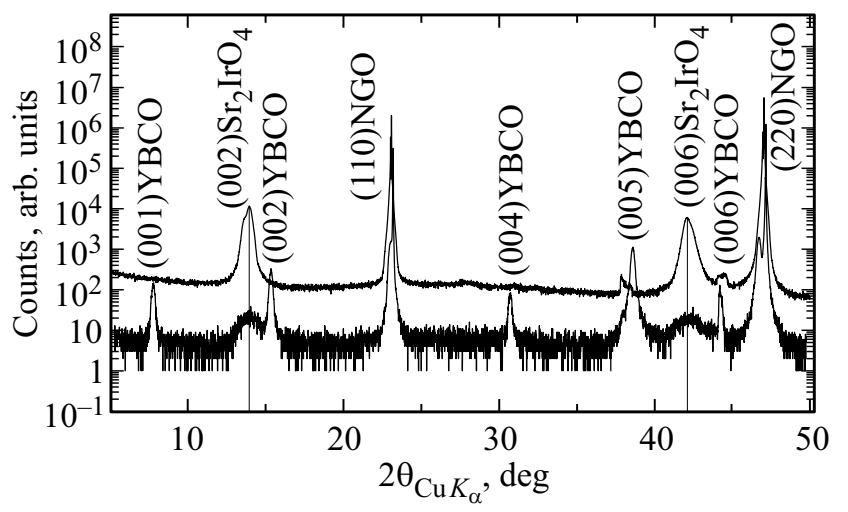

Рис. 6. Рентгеновские дифрактограммы, снятые в режиме брегговского отражения, для автономной пленки $\mathrm{Sr}_{2} \mathrm{IrO}_{4}$ толщиной $20 \mathrm{~nm}$, выращенной на подложке из $\mathrm{NdGaO}_{3}$ (верхний спектр) и гетероструктуры $\mathrm{Au} / \mathrm{Sr}_{2} \mathrm{IrO}_{4} / \mathrm{YBa}_{2} \mathrm{Cu}_{3} \mathrm{O}_{x}$ полученной также на подложке $\mathrm{NdGaO}_{3}$ (нижний спектр). Толщина пленки золота $\mathrm{Au}-10 \mathrm{~nm}, \mathrm{Sr}_{2} \mathrm{IrO}_{4}-10 \mathrm{~nm}, \mathrm{YBa}_{2} \mathrm{Cu}_{3} \mathrm{O}_{x}-60 \mathrm{~nm}$. 
дифрактограммы в режиме бреговского отражения для пленки $\mathrm{Sr}_{2} \mathrm{IrO}_{4}$, выращенной на подложке $\mathrm{NdGaO}_{3}$ и гетероструктуры $\mathrm{Au} / \mathrm{Sr}_{2} \mathrm{IrO}_{4} / \mathrm{YBa}_{2} \mathrm{Cu}_{3} \mathrm{O}_{x}$. Пленка $\mathrm{YBCO}$ растет на подложке NGO эпитаксиально с осью $c$ перпендикулярной плоскости подложки, что подтверждают снятые „косые срезы“. Пленка $\mathrm{Sr}_{2} \mathrm{IrO}_{4}$, выращенная на YBCO, также $c$-ориентирована. Кристаллические параметры пленки $\mathrm{Sr}_{2} \mathrm{IrO}_{4}$, напыленной поверх оказались близки к значениям для автономной пленки (см. рис. 6). Гетероструктуры покрывались сверху пленкой ниобия с помощью магнетронного распыления в атмосфере аргона при комнатной температуре.

Снималась температурная зависимость гетероструктуры до напыления ниобия и после при токе, протекающим в плоскости подложки. Было обнаружено уменьшение критической температуры сверхпроводящего перехода от $90 \mathrm{~K}$ сразу после изготовления гетероструктуры до $80-85 \mathrm{~K}$ поле после напыления пленки $\mathrm{Nb}$ и проведенного ионного травления через фотомаску, формирующую геометрию нижнего электрода.

Мезаструктуры микронных размеров от $10 \times 10$ до $50 \times 50 \mu \mathrm{m}^{2}$ формировались с помощью оптической литографии, реактивного ионно-плазменного и ионно-лучевого травлений при низких ускоряющих напряжений. Пленка $\mathrm{SiO}_{2}$, которая выполняла функции межслойного изолятора и обеспечивала протекание тока перпендикулярно слоям, напылялась радиочастотным распылением. Дополнительно напыленный слой $\mathrm{Nb}$ толщиной $200 \mathrm{~nm}$ обеспечивал сверхпроводящие подводы тока. Контактные площадки были выполнены из золота. Использовалась четырехконтактная схема подключения к мезаструктуре: два контакта (для тока и напряжения) к YВСО электроду и два к электроду из $\mathrm{Nb}$ (см. рис. 7). Эффект близости между $\mathrm{Nb}$ и Аu обеспечивал проникновение сверхпроводящего параметра порядка к границе $\mathrm{Au} / \mathrm{Sr}_{2} \mathrm{IrO}_{4}[26]$.

Температурные зависимости сопротивления мезаструктур имели активационный характер также как и у автономных пленок $\mathrm{Sr}_{2} \mathrm{IrO}_{4}$ (сравни рис. 4 и 8). Эти данные указывают на то, что проводимость мезаструктур имеет туннельный характер.

Измерялась BAX мезаструктур при температуре жидкого гелия $(T=4.2 \mathrm{~K})$ для двух диапазонов измерения напряжения \pm 10 и $\pm 100 \mathrm{mV}$. Дифференциальное сопротивление и проводимость определялись из дифференцирования полученных цифровых данных. Для оценки параметров туннельного барьера использовался известный метод измерения зависимости проводимости мезаструктуры от напряжений, сравнимых с высотой барьера $[27,28]$, при больших напряжений. Сначала по величине проводимости мезаструктуры при малых напряжениях $G(0)$ определялась величина высоты барьеpa $\varphi($ в $\mathrm{eV})$ в предположении симметричного барьера толщиной $d$ прослойки из $\mathrm{Sr}_{2} \mathrm{IrO}_{4}[28]$

$$
G(0)=\left(3.16 \cdot 10^{10} \varphi^{1 / 2} / d\right) \exp \left(-1.025 d \varphi^{1 / 2}\right),
$$

используя экспериментальные значения $G(0)$ измеренного в $\Omega^{-1}$ и $d$ в измеренного в Ангстремах. Асиммет-

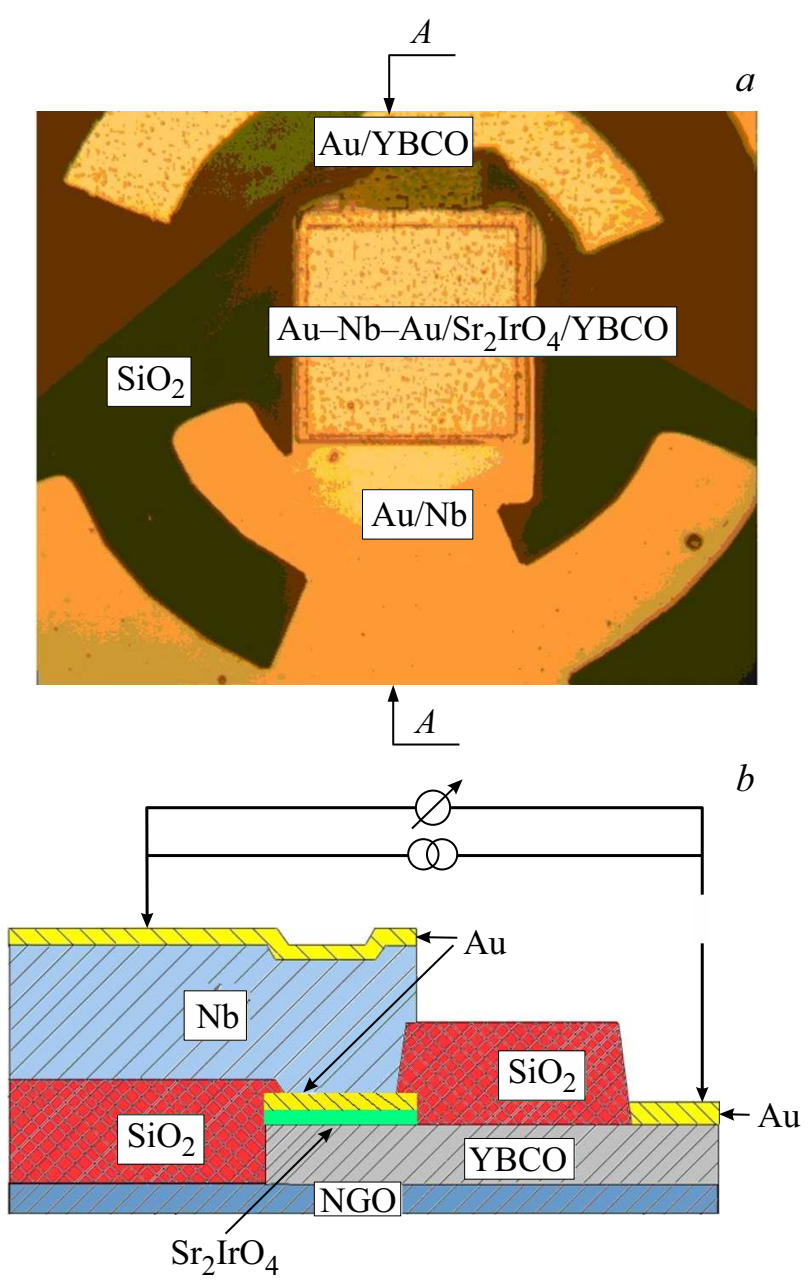

Рис. 7. Вид сверху и поперечное сечение мезаструктуры. $\mathrm{SiO}_{2}$ - изолятор, используемый для предотвращения транспорта тока в мезаструктуре вдоль плоскости подложки. Задание тока и измерение напряжения осуществляется через контакты из золота $\mathrm{Au}$.

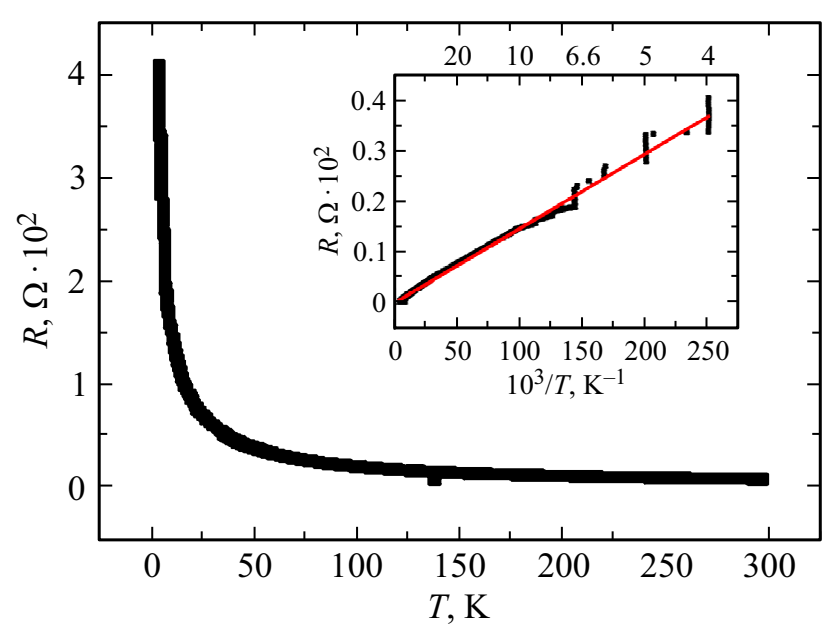

Рис. 8. Температурная зависимость сопротивления мезаструктуры размером $10 \times 10 \mu \mathrm{m}^{2}$ и толщиной прослойки $10 \mathrm{~nm}$. На вставке показана температурная зависимость характерного сопротивления границы от обратной температуры. 


\begin{tabular}{c|c|c|c|c|c|c|c}
\hline$N$ & $A, \mu \mathrm{m}^{2}$ & $d, \mathrm{~nm}$ & $\varphi, \mathrm{V}$ & $d \varphi, \mathrm{V}$ & $R_{0}, \Omega$ & $R_{0} A \mu \Omega \cdot \mathrm{cm}^{2}$ & $\Delta G / G(0)$ \\
\hline $07 \mathrm{Ne}$ & $10 \times 10$ & 5 & 0.24 & 0.19 & 256 & 256 & 0.277 \\
$07 \mathrm{Nd}$ & $20 \times 20$ & 5 & 0.25 & 0.17 & 503 & 2012 & 0.227 \\
$07 \mathrm{Nb}$ & $40 \times 40$ & 5 & 0.24 & 0.17 & 322 & 5152 & 0.355 \\
$08 \mathrm{Ne}$ & $10 \times 10$ & 7 & 0.11 & 0.15 & 182 & 832 & 0.175 \\
$09 \mathrm{Ne}$ & $10 \times 10$ & 10 & 0.08 & 0.3 & 83521 & 83521 & 0.398
\end{tabular}

Примечание. $A-$ размер мезаструктуры в плане, $d-$ толщина прослойки, $\varphi$ и $\Delta \varphi-$ среднее значение высоты туннельного барьера и его асимметрия, соответственно, полученным из подгонки зависимости $G(V)$ по формулам работ $[27,28], R_{0}-$ дифференциальное сопротивление мезаструктуры при малых напряжениях, $\Delta G / G(0)$ - нормированое значение скачка проводимости мезаструктуры.

рия зависимости $G(V)$ указывает на асимметрию туннельного барьера (см. рис. 9, $a$ ). Из значения напряжения, соответствующего минимуму $G(V)$, определялась асимметрия барьера $\Delta \varphi$

$$
e V_{\min }=0.649\left(\Delta \varphi / d \varphi^{1 / 2}\right)
$$

Зависимость $G(V)$ определялась по следующей формуле [27]:

$$
G(V) / G(0)=1-\left(A_{0} \Delta \varphi / 16 \varphi^{3 / 2}\right) e V+\left(9 A_{0}^{2} / 128 \varphi\right)(e V)^{2},
$$

где параметр $A_{0}=4(2 m)^{1 / 2} d / 3 h$ определяется толщиной барьера $d$. На рис. 9, $a$ для образца $08 \mathrm{Ne}$ показаны экспериментальная зависимость и определенная по формуле (4) при определенных по (2) и (3) значениям $d, \varphi$ и $\Delta \varphi$ и зависимость при параметрах барьера, дающих наилучшее соответствие с экспериментом. Результаты
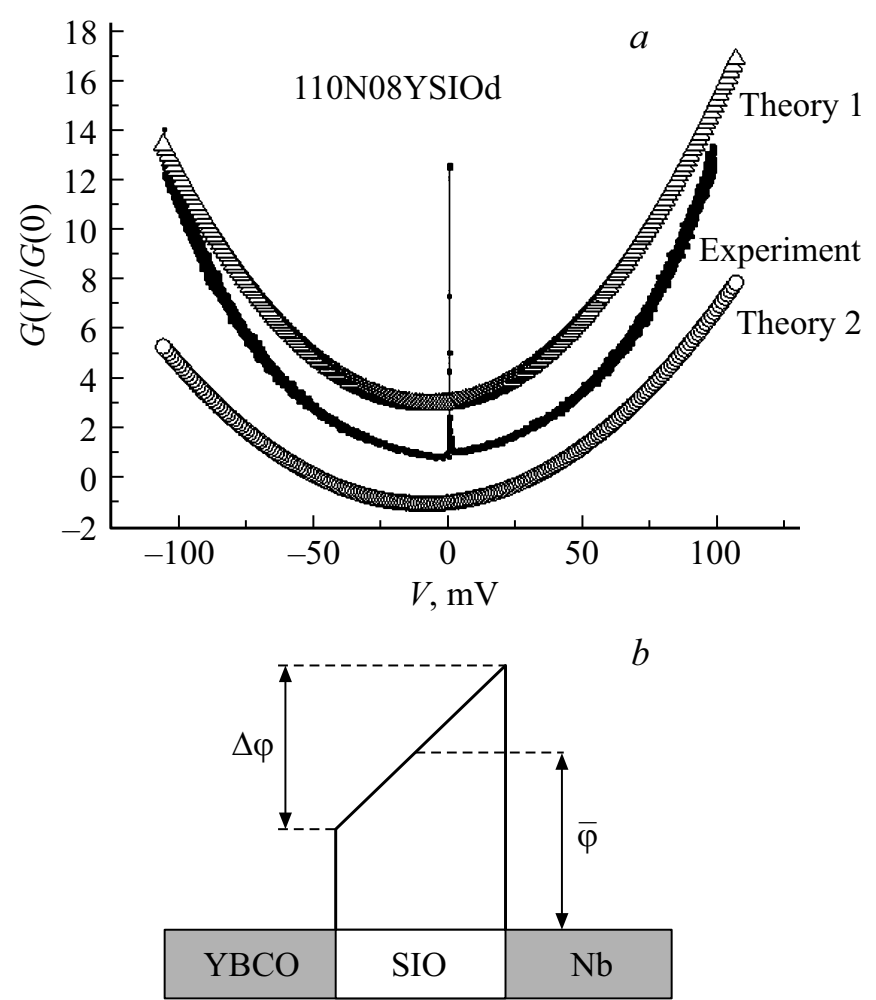

Рис. 9. $a-$ зависимость проводимости мезаструктуры $\mathrm{Nb}-\mathrm{Au} / \mathrm{Sr}_{2} \mathrm{IrO}_{4} / \mathrm{YBa}_{2} \mathrm{Cu}_{3} \mathrm{O}_{x}$ размером $10 \times 10 \mu \mathrm{m}^{2}$ от напряжения; $b-$ схема барьера гетероструктуры.

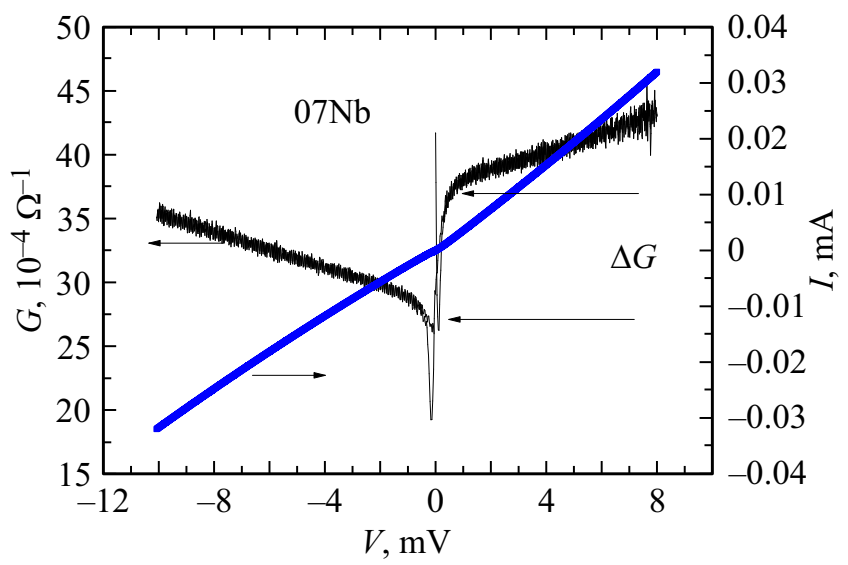

Рис. 10. Скачок проводимости и ВАХ мезаструктуры при низких напряжениях $(V<10 \mathrm{mV})$.

измерений пяти образцов, где наблюдались аналогичные зависимости $G(V)$ представлены в таблице. На наших мезаструктурах на зависимостях $G(V)$ при малых напряжениях наблюдались особенности, которые не описываются туннельной моделью [27,28]. При напряжениях близких к нулю $(V \ll 1 \mathrm{mV})$ наблюдался скачок проводимости $\Delta G / G(0)$ величиной $20-30 \%$ (рис. 10). Он не наблюдается в мезаструктурах с прослойкой из манганита, хотя слабая асимметрия проводимости $G(V)$ была обнаружена в обоих структурах [29]. Возможно наблюдаемый скачок $\Delta G / G(0)$ вызван наличием в прослойке сильного спин-орбитального взаимодействия.

\section{5. Заключение}

Разработана технология лазерной абляции эпитаксиальных тонких пленок семейства Ruddlesden-Popper оксидов иридия $\left(\mathrm{Sr}_{n+1} \mathrm{Ir}_{n} \mathrm{O}_{3 n+1} ; n=1,2\right.$, и $\left.\infty\right)$ : парамагнитный плохой метал $\mathrm{SrIrO}_{3}$ и антиферромагнитный изолятор $\mathrm{Sr}_{2} \mathrm{IrO}_{4}$. Рост эпитаксиальных пленок удается осуществить из одной и той же мишени $\mathrm{Sr}_{2} \mathrm{IrO}_{4}$, изменяя давление в камере активного газа при росте. Температурные зависимости сопротивления пленок $\mathrm{Sr}_{2} \mathrm{IrO}_{4}$ имели активационную зависимость, в то время как сопротивление пленок $\mathrm{SrIrO}_{3}$ слабо зависело от температуры и имело переход металл-изолятор при температуре около $100 \mathrm{~K}$. Были изготовлены мезаструк- 
туры микронных размеров из сверхпроводящих гетероструктур $\mathrm{Nb}-\mathrm{Au} / \mathrm{Sr}_{2} \mathrm{IrO}_{4} / \mathrm{YBa}_{2} \mathrm{Cu}_{3} \mathrm{O}_{x}$ с прослойкой 5, 7 и $10 \mathrm{~nm}$. Используя модель туннельного перехода удалось оценить высоту и асимметрию туннельного барьера в мезаструктуре. Наблюдался скачок проводимости мезаструктуры при малых напряжениях, который не удается объяснить в рамках известных моделей туннельных структур. Возможно такое необычное поведение проводимости вызвано наличием сильного спин-орбитального взаимодействия в прослойке из иридата.

\section{Список литературы}

[1] S.J. Moon, H. Jin, K.W. Kim, W.S. Choi, Y.S. Lee, J. Yu, G. Cao, A. Sumi, H. Funakubo, C. Bernhard, T.W. Noh. Phys. Rev. Lett. 101, 226402 (2008).

[2] W. Witczak-Krempa, G. Chen, Y.B. Kim, L. Balents. Ann. Rev. Condens. Matter. Phys. 5, 57 (2014).

[3] R. Schaffer, E. Lee, B. Yang, Y. Kim. Rep. Prog. Phys. 79, 094504 (2016).

[4] A. Shitade, H. Katsura, J. Kuneš, X.-L. Qi, S.-C. Zhang, N. Nagaosa. Phys. Rev. Lett. 102, 256403 (2009).

[5] D. Xiao, W. Zhu, Y. Ran, N. Nagaosa, S. Okamoto. Nature Commun. 2, 596 (2011).

[6] F. Wang, Y. Ran. Phys. Rev. B 84, 241103 (2011).

[7] X. Wan, A.M. Turner, A. Vishwanath, S.Y. Savrasov. Phys. Rev. B 83, 205101 (2011).

[8] J.G. Kim, D. Casa, M.H. Upton, T. Gog, Y.-J. Kim, J.F. Mitchell, M. van Veenendaal, M. Daghofer, J. van den Brink, G. Khaliullin, B.J. Kim. Phys. Rev. Lett. 108, 177003 (2012).

[9] B.J. Kim, H. Ohsumi, T. Komesu, S. Sakai, T. Morita, H. Takagi, T. Arima. Science 323, 1329 (2009).

[10] G. Cao, Y. Xin, C.S. Alexander, J.E. Crow, P. Schlottmann, M.K. Crawford, R.L. Harlow, W. Marshall. Phys. Rev. B 66, 214412 (2002).

[11] M. Longo, J.A. Kafalas, R.J. Arnott. J. Solid State Chem. 3, 174 (1971).

[12] A.M. Петржик, G. Cristiani, Г. Логвенов, А.Е. Пестун, Н.В. Андреев, Ю.В. Кислинский, Г.А. Овсянников. Письма в ЖТФ 43, 25 (2017).

[13] C. Lu, A. Quindeau, H. Deniz, D. Preziosi, D. Hesse, M. Alexe. Appl. Phys. Lett. 105, 082407 (2014).

[14] Perovskite Materials-Synthesis, Characterisation, Properties, and Applications / Eds. Likun Pan and Guang Zhu. InTech, Chapters (2016). 648 p.

[15] Feng Ye, Songxue Chi, Bryan C. Chakoumakos, Jaime A. Fernandez-Baca, Tongfei Qi, G. Cao. Phys. Rev. B 87, 140406(R) (2013).

[16] X. Liu, Y. Cao, B. Pal, Middey, M. Kareev, Y. Choi, P. Shafer, D. Haskel, E. Arenholz, J. Chakhalian. Phys. Rev. Mater, 1, 075004 (2017).

[17] Ю.В. Кислинский, Г.А. Овсянников, А.М. Петржик, К.И. Константинян, Н.В. Андреев, Т.А. Свиридова. ФТТ 57, 2446 (2015).

[18] J. Nichols, J. Terzic, E.G. Bittle, O.B. Korneta, L.E. De Long, J.W. Brill, G. Cao, S.S.A. Seo. Appl. Phys. Lett. 102, 141908 (2013).

[19] J.H. Gruenewald, J. Nichols, J. Terzic, G. Cao, J.W. Brill, S.S. Ambrose. J. Mater. Res. 29, 2491 (2014).
[20] F.X. Wu, J. Zhou, L.Y. Zhang, Y.B. Chen, S.T. Zhang, Z.B. Gu, S.H. Yao, Y.F. Chen. J. Phys.: Condens. Matter 25, 125604 (2013).

[21] G. Zhao, L.X. Yang, Y. Yu, F.Y. Li, R.C. Yu, Z. Fang, L.C. Chen, C.Q. Jina. J. Appl. Phys. 103, 103706 (2008).

[22] A. Biswas, Ki-Seok Kim, Yoon Hee Jeong. J. Appl. Phys. 116, 213704 (2014).

[23] P.A. Lee, T.V. Ramakrishnan. Rev. Mod. Phys. 57, 287 (1985).

[24] G.R. Stewart. Rev. Mod. Phys. 73, 797 ( 2001).

[25] Y.S. Lee, J.S. Lee, K.W. Kim, T.W. Noh, J. Yu, E.J. Choi, G. Cao, J.E. Crow. Europhys. Lett. 55, 280 (2001).

[26] P. Komissinskiy, G.A. Ovsyannikov, K.Y. Constantinian, Y.V. Kislinski, I.V. Borisenko, I.I. Soloviev, V.K. Kornev, E. Goldobin, D. Winkler. Phys. Rev. B 78, 024501 (2008).

[27] W.F. Brinkman, R.C. Dynes, J.M. Rowell. J. Appl. Phys. 41, 1915 (1970).

[28] John G. Simmons. J. Appl. Phys. 34, 1793 (1963).

[29] Г.А. Овсянников, Ю.В. Кислинский, К.И. Константинян, А.В. Шадрин, В.В. Демидов, А.М. Петржик. ЖЭТФ 151, 736 (2017).

Редактор К.В. Емцев 